\title{
A LATTICE HOMOMORPHISM OF A LATTICE ORDERED GROUP ${ }^{1}$
}

\section{CASPER GOFFMAN}

The homomorphism we have in mind has been introduced by Jaffard, [1], ${ }^{2}$ for lattice ordered groups, and has been discussed by Pierce, [2], for any distributive lattice $L$ with a minimal element.

It may be defined as the homomorphism $\alpha$ of $L$ for which $\alpha(x)$ $=\alpha(y)$ if and only if the set of elements in $L$ disjoint with $x$ is the same as the set disjoint with $y$. Pierce has shown that this is the maximal lattice homomorphism of $L$ whose kernel is [0], the set whose only element is 0 , and that it is the only lattice homomorphism of $L$, with kernel [0], whose image is disjunctive. Jaffard called the elements of the image lattice (i.e., the equivalence sets of the homomorphism) filets in $L$, but we prefer the term carriers in $L$ because of their role in function spaces.

Our purpose is to obtain a characterization of $\alpha$, which involves the group operation, for the case where $L$ is the positive cone of an archimedean lattice ordered group.

Jaffard has shown, in this case, that $\alpha$ has the following properties:

(a) $a \leqq b$ implies $\alpha(a) \leqq \alpha(b), a>0$ implies $\alpha(a)>\alpha(0)$.

(b) $\alpha$ is a lattice homomorphism; i.e., $\alpha(a \cup b)=\alpha(a) \cup \alpha(b), \alpha(a \cap b)$ $=\alpha(a) \cap \alpha(b)$.

(c) $\alpha(a+b)=\alpha(a \cup b)$.

We show that $\alpha$ has the additional property:

(d) If $x=\sup \left[x_{i} \mid i \in I\right]$, then sup $\left[\alpha\left(x_{i}\right) \mid i \in I\right]$ exists and $\alpha(x)$ $=\sup \left[\alpha\left(x_{i}\right) \mid i \in I\right]$.

Proof. First, $\alpha(x) \geqq \alpha\left(x_{i}\right)$ for all $i \in I$. Suppose there is $z>0$ such that $\alpha(z) \geqq \alpha\left(x_{i}\right)$, for all $i \in I$, but $\alpha(z)<\alpha(x)$. By the disjunctive property of the lattice of carriers, there is a $w>0$ such that $\alpha(w) \leqq \alpha(x)$ and $\alpha(w) \cap \alpha(z)=\alpha(0)$. Then $\alpha(w) \cap \alpha\left(x_{i}\right)=\alpha(0)$, for all $i \in I$. Let $v=x \cap w$. Then $\alpha(v)=\alpha(x) \cap \alpha(w)=\alpha(w)$, and $v>0$. It follows that $v \cap x_{i}=0$, for all $i \in I$. Now, $v \leqq x, x_{i} \leqq x$, and $v \cap x_{i}=0$ implies that $v+x_{i}=v \cup x_{i} \leqq x$ for all $i \in I$. If we let $y=x-v$, then $y<x$ and $y \geqq x_{i}$ for all $i \in I$. But this contradicts the definition of $x$.

Presented to the Society, November 23, 1956; received by the editors September 12, 1956.

1 The work, of which this note is a part, is being sponsored by National Science Foundation grant no. NSF G-2267.

2 The numbers in brackets refer to the references at the end of the paper. 
We now show that $\alpha$ is the only lattice homomorphism with zero kernel of the positive cone $L$ of an archimedean lattice ordered group $G$ which has properties (c) and (d). For, suppose $\beta$ has these properties: We use the following:

Lemma. If $\alpha(x)=\alpha(y)$ and $0<x \leqq y$, then

$$
y=\sup (n x \cap y) \text {. }
$$

Proof. Otherwise, there is $w<y$ such that $n x \cap y \leqq w$, for all $n$. Let $z=y-w$ and let $u=z \cap x$. Then $\alpha(u)=\alpha(z) \cap \alpha(x)=\alpha(z)>\alpha(0)$, so that $u>0$. But $n u<y$, for every $n$. This contradicts the assumption that $G$ is archimedean.

We suppose first that $0<x \leqq y$ and $\alpha(x)=\alpha(y)$. Now, by (c), (and this is the only implication of (c) we need), $\beta(n x)=\beta(x)$ for every $x$. Since $x \leqq n x \cap y \leqq n x$, we then have $\beta(n x \cap y)=\beta(x)$. Now, by (d), and the lemma, we have $\beta(x)=\beta(y)$.

We now consider the general case $x>0, y>0$ and $\alpha(x)=\alpha(y)$. Then $\alpha(x \cap y)=\alpha(x)=\alpha(y)$ so that, since $0<x \cap y \leqq x$, we have $\beta(x)$ $=\beta(x \cap y)$. Similarly, $\beta(y)=\beta(x \cap y)$. Hence $\alpha(x)=\alpha(y)$ always implies $\beta(x)=\beta(y)$. Since $\alpha$ is the maximal lattice homomorphism with kernel [0], it follows that $\alpha$ and $\beta$ are the same. Hence, we have the:

THEOREM. If $G$ is an archimedean lattice ordered group with positive cone $L$, then there is a unique supremum preserving lattice homomorphism $\alpha$ of $L$, with kernel [0], such that

$$
\alpha(x \cup y)=\alpha(x+y)
$$

for all $x, y \in L$, and the image of $L$ under $\alpha$ is the lattice of carriers in $L$.

We now give several examples. The first shows that the theorem does not always hold in nonarchimedean groups.

1. We consider the totally ordered group $G$ of pairs $(a, b)$ of real numbers, ordered lexicographically. Then $G$, as a totally ordered group with more than one element, has 2 carriers. We consider also a totally ordered set of 3 elements $\xi<\eta<\zeta$, and the homomorphism $\beta$ of the positive cone $L \subset G$ defined by

$$
\begin{array}{ll}
\beta(0,0)=\xi, & \\
\beta(0, b)=\eta & \text { if } b>0, \\
\beta(a, b)=\zeta & \text { if } a>0 .
\end{array}
$$

It is an easy matter to verify that $\beta$ satisfies conditions (a), (b), (c), and (d).

The next three examples are concerned with the independence of 
conditions (c) and (d) of each other and of the lattice homomorphism conditions.

2. Let $G$ be the totally ordered group of real numbers and let $\beta$ be the lattice homomorphism defined as follows:

$$
\begin{array}{lr}
\beta(0)=0, & \\
\beta(x)=1 & \text { for } 0<x<2, \\
\beta(x)=n & \text { for } n \leqq x<n+1, n=2,3, \cdots
\end{array}
$$

Then $\beta$ is evidently a lattice homomorphism. But it satisfies neither conditions (c) nor (d). For, if $S$ is the set of all $x<2$ then sup $[\beta(x) \mid x$ $\in S]=1$, but $\beta(\sup S)=2$, so that $\beta$ does not satisfy condition (d). That it does not satisfy condition (c) is obvious.

3 . Let $G$ be any lattice ordered group which is not totally ordered; say, the real functions on $[0,1]$, and let $\beta$ be the identity homomorphism. Then $\beta$ satisfies condition (d) but evidently not condition (c).

4. Let $G$ again be the lattice ordered group of all real functions on $(0,1)$, and let $\beta(x)=\beta(y)$ if and only if $\alpha(x)=\alpha(y)$ and $x$ and $y$ are either both bounded or both unbounded functions. The order relation is $\beta(x) \geqq \beta(y)$ if either

$$
\begin{aligned}
& \alpha(x) \geqq \alpha(y) \text { and } x \text { and } y \text { are both bounded, or } \\
& \alpha(x) \geqq \alpha(y) \text { and } x \text { is unbounded. }
\end{aligned}
$$

That $\beta$ is a lattice homomorphism is easy to verify.

That $\beta$ does not satisfy (d) is shown by letting

$$
\begin{aligned}
y_{n}=y_{n}(t) & =n & & \text { for } 0<x<2^{-n+1} \\
& =0 & & \text { for } 2^{-n+1}<x<1,
\end{aligned}
$$

and

$$
x_{n}=x_{n}(t)=\sum_{m=1}^{n} y_{m}(t), \quad n=1,2, \cdots
$$

It is clear, in this case, that $\beta\left(\sup x_{n}\right) \neq \sup \beta\left(x_{n}\right)$.

Finally, that $\beta$ satisfies (c) follows from the fact that $\alpha$ satisfies this condition and that if $x$ and $y$ are both bounded then $x \cup y$ and $x+y$ are both bounded, while if at least one of $x$ and $y$ is unbounded then both $x \cup y$ and $x+y$ are unbounded.

Examples 2, 3, and 4 show that a lattice homomorphism of an archimedean lattice ordered group can either satisfy neither conditions (c) nor (d), (c) but not (d), or (d) but not (c). 
The last example is of a distributive lattice with minimal element 0 for which the homomorphism $\alpha$ itself does not satisfy condition (d).

5. Let $L$ be the lattice of real functions defined on an uncountable set $S$ which assume the values 0,1 , or 2 , but can have the value 2 only on a finite set or else everywhere. $L$ is ordered in the usual way for functions. The lattice of carriers in $L$ may be identified with the lattice of subsets of $S$, the carrier of $f \in L$ being the set on which $f$ does not vanish. We consider a countable set $\left\{f_{n}\right\}$ of functions in $L$, each of which has the value 2 at a single point $x_{n} \in S$ and is 0 everywhere else. The carrier of $f_{n}$ is then $\left[x_{n}\right]$, the set whose only element is $x_{n}$. Now, $\sup f_{n}=f$, where $f$ is the function which is 2 everywhere. The carrier of $f$ is $S$. But $S \neq$ sup $\left[x_{n}\right]$.

As a final remark, we note that in the proof of the theorem we used the weaker properties:

(c') $\beta(n a)=\beta(a)$.

$\left(\mathrm{d}^{\prime}\right)$ If $\beta\left(x_{i}\right)=\beta(y)$ for all $i \in I$ and if $x=\sup \left[x_{i} \mid i \in I\right]$ then $\sup \left[\beta\left(x_{i}\right) \mid i \in I\right]$ exists and is equal to $\beta(x)$.

This implies that $\left(\mathrm{c}^{\prime}\right)$ and $\left(\mathrm{d}^{\prime}\right)$ are equivalent to $(\mathrm{c})$ and $(\mathrm{d})$.

\section{REFERENCES}

1. P. Jaffard, Contribution d l'étude des groupes ordonnés, Journal de Mathématiques Pures et Appliquées vol. 32 (1953) pp. 203-280.

2. R. S. Pierce, Homomorphisms in semi-groups, Ann. of Math. vol. 59 (1954) pp. 287-291.

UNIVERSITY OF OKLAHOMA 